\title{
Deficits in Social Behavior Precede Cognitive Decline in Middle-Aged Mice
}

\author{
Flora Boyer, Florence Jaouen, El Chérif Ibrahim and Eduardo Gascon* \\ Aix Marseille Univ, CNRS, Institut de Neurosciences de la Timone (INT), Marseille, France
}

An extensive literature details deterioration of multiple brain functions, especially memory and learning, during aging in humans and in rodents. In contrast, the decline of social functions is less well understood. It is presently not clear whether age-dependent deficits observed in social behavior mainly reflect the disruption of social networks activity or are simply secondary to a more general impairment of cognitive and executive functions in older individuals. To address this issue, we carried out a battery of behavioral tasks exploring different brain functions in young ( 3 months) and middle-aged wild-type mice (9 months). Consistent with previous reports, our results show no obvious differences between these two groups in most of the domains investigated including learning and memory. Surprisingly, in social tasks, middle-aged animals showed significantly reduced levels of interactions when exposed to a new juvenile mouse. In the absence of overt cognitive decline, our findings suggest that social impairments may precede the disruption of other brain functions and argue for a selective vulnerability of social circuits during aging.

Donald A. Wilson,

New York University,

United States

Reviewed by:

Ramon Velazquez, Arizona State University,

United States

Frédérique Rene,

Université de Strasbourg, France

*Correspondence:

Eduardo Gascon eduardo.gascon-gonzalo@univamu.fr

Received: 25 January 2019 Accepted: 06 March 2019 Published: 27 March 2019

Citation: Boyer F, Jaouen F, Ibrahim EC and Gascon E (2019) Deficits in Social Behavior Precede Cognitive Decline in Middle-Aged Mice. Front. Behav. Neurosci. 13:55. doi: 10.3389/fnbeh.2019.00055
Keywords: social behavior, normal aging, cognitive impairment, neurodegeneration, mice

\section{INTRODUCTION}

Although all organs and tissues in an organism deteriorate with age, the brain is particularly sensitive to the aging process. The anatomical organization of the brain and the unusual characteristics of neuronal physiology explain this selective vulnerability (for review see Bishop et al., 2010).

Functionally, aging has been consistently associated with impairments in different cognitive domains in humans and also in animal models (McQuail et al., 2015; Wyss-Coray, 2016; Sadoun et al., 2019). Although social activities are prominent in humans (van Schaik et al., 2012), whether these brain functions also decline with age has not been examined until recently. Impairments in certain social functions such as recognition of mental states or emotions in others (theory of mind) have been observed in aged people (for review see Moran, 2013; Fortier et al., 2016). Nonetheless, there are conflicting interpretations of these results; some studies suggest that deficits in social tasks might be, at least in part, the consequence of a more generalized age-related impairment in brain activity, namely in working memory, attention and/or executive functions (Bottiroli et al., 2016; Johansson Nolaker et al., 2018). In contrast, others seem to indicate that aging elicits a primary decline of social capabilities (Moran et al., 2012; Cavallini et al., 2013).

Similarly, behavioral studies in rodents have also shown a decrease in social interactions (Salchner et al., 2004) or social recognition memory with age (Prediger et al., 2005; 
Markham and Juraska, 2007). In the same line, it has been reported that older female macaques spend less time in close proximity to others and engage in fewer social interactions (Corr, 2003; Almeling et al., 2016, 2017). Together, these observations indicate that animal models can reproduce some of the age-related social cognitive deficits in humans and can help to better understand the neural correlates of such a decline.

Here, we have questioned whether aging can affect social functions in the first place. To address this problem, we compared the behavioral performance of young (3 months) and middle-aged (9 months) mice in different social and non-social tasks. We have selected this latter point over time because it has already been shown to be before the onset of cognitive decline (Lamberty and Gower, 1990; Frick et al., 1995; Bishop et al., 2010; Bizon et al., 2012). Accordingly, our results indicate that middle-aged mice have no cognitive impairments. Interestingly, animals show a significant reduction in social drive but other domains of social cognition, such as social exploration or social memory are spared. Together, these observations support the notion that certain social circuits may be particularly vulnerable to aging and that social deficits may be early symptoms of brain aging.

\section{MATERIALS AND METHODS}

\section{Animals}

Mice were purchased from Charles Rivers Laboratories (France). A total of $48 \mathrm{C} 57 \mathrm{Bl6} / \mathrm{J}$ male mice were used in this study. Half of them were young adults $(n=24,10$ weeks at the time of arrival) whereas the other animals were older adults $(n=24$, 9 months). Upon arrival, each male was housed with an elderly female presenting a low fertility (>12 months) for 2 weeks. This enables acclimation to the animal facility and avoids the harmful effects of isolation while limiting reproduction and fighting. The animals were kept under specific pathogen-free conditions with a regular 12-h light/dark cycle (light on at 8:00 am) and constant conditions $\left(21 \pm 1^{\circ} \mathrm{C} ; 50 \%\right.$ humidity). Food and water were supplied ad libitum. Mice also benefited from some environmental enrichment (a wooden stick and a piece of cotton with each cage change).

All procedures involving mice were approved by the local ethics committee (EU0488, \#6357) and are in agreement with European regulations (Directive 2010/63/EU). A special effort was made in handling animals to minimize stress or anxiety.

\section{Behavioral Schedule and Exclusion Criteria}

All experiments were conducted between 9 am and $7 \mathrm{pm}$. Animals were habituated to the behavioral room for at least $30 \mathrm{~min}$ before the start of the task. The 48 animals to be analyzed were divided into three cohorts that were tested independently. For each cohort of animals, tests were conducted according to this schedule: (1) activity monitoring (Day 1); (2) three-chamber social task (Day 7); (3) intruder test (Day 9); (4) interactions in neutral arena (Day 11); (5) open field and novel object recognition (Day 13); (6) Morris water maze (MWM; Day 15-19); and (7) olfactory behavior (Day 21). We randomized the order of animals on each test.
One middle-aged animal with severe distress signs at the end of the activity monitoring was euthanized and therefore excluded from the study. Due to a software error during acquisition, it was not possible to record a video of a 3-month-old animal in the novel object recognition task. Consequently, this animal was excluded from the analysis for this task only. In the olfactory testing, trials in which an animal frequently moved the cotton were excluded from the analysis.

\section{Activity Monitoring}

To obtain an overview of basic behavior (locomotion, feeding, drinking, resting. . .), mice were tracked for $20 \mathrm{~h}$ in an activity box (PhenoTyper, Noldus, Netherlands). Briefly, mice were placed in a dedicated cage $(30 \times 30 \times 38 \mathrm{~cm})$ containing the litter from their cage. At the top of the cage, an instrumented unit equipped with an infrared sensitive camera and three arrays of infrared LED lights enabled video recording and analysis regardless of ambient light conditions. Four PhenoTyper devices were used in parallel in this study. To avoid visual interference between animals running the test simultaneously, black curtains surrounded each PhenoTyper.

\section{Open Field}

The open field test is a straightforward test to study locomotion and exploratory behavior in mice. The open field device consisted of a non-reflective opaque plexiglass box $(40 \times 40 \times 40 \mathrm{~cm})$ and a suspended digital camera. The animals were placed in facing an open field wall and their behavior was recorded for $10 \mathrm{~min}$. The total distance covered, the time spent in the center and near the walls were calculated.

\section{Novel Object Recognition}

For the novel object recognition task, we used the open field device and small plastic objects. In the first phase, the animals were placed facing a wall and two identical objects (green cylinders, $3.5 \mathrm{~cm}$ high, $4.5 \mathrm{~cm}$ in diameter) were placed in front of the opposite wall of the arena $(10 \mathrm{~cm}$ from the wall and $5.5 \mathrm{~cm}$ apart from each other). The mice explored the arena for $10 \mathrm{~min}$ and were brought back to their cages for $10 \mathrm{~min}$. In the second phase, one of the objects was replaced by a novel object (yellow triangular prism, $3.5 \mathrm{~cm}$ high, $4.5 \mathrm{~cm}$ on each side). The time spent exploring each object (nose point within $2 \mathrm{~cm}$ from the object) was quantified and used to calculate a Recognition Index (RI) as follows: $\mathrm{RI}=($ time exploring object 1$) /($ time exploring object $1+$ time exploring object 2 ).

\section{Morris Water Maze}

The MWM was performed as previously described with minor modifications (LaSarge et al., 2007). Briefly, a round pool $(120 \mathrm{~cm}$ in diameter) was filled with water $\left(25^{\circ} \mathrm{C}\right)$ and tempera paint was added to the water until it becomes opaque. A hidden platform (10 $\mathrm{cm}$ in diameter) was placed about $1 \mathrm{~cm}$ below the water surface. The spatial cues consisted of geometric figures of different colors located in privacy blinds surrounding the water tank. The subjects were recorded by a video tracking system directly above the water tank.

During the training phase ( 4 days) each animal received three trials. In each trial, the animal was placed in the water tank 
facing the wall and allowed to explore the maze until it reached the platform. If the animal did not find the platform in $2 \mathrm{~min}$, the experimenter guided it to the platform. In either case, the animal was left on the platform for $10 \mathrm{~s}$. Then it was dried and returned to the cage for $5 \mathrm{~min}$ until the next trial. The entry point was randomized for each animal and each training day but was the same for the three trials an animal performed over a training session. Once all the animals had completed the training phase, they each performed one probe trial $(2 \mathrm{~min})$ on day 5 , during which the platform was removed from the pool. The probe trial was performed to verify the animal's understanding of the platform location and to observe the exploratory strategy it followed when it discovered that the platform had been removed. The latency to reach the platform and the frequency of crossings were evaluated.

\section{Olfactory Testing}

This is a modification of the olfactory habituation/dishabituation test (Moreno et al., 2014) and consists of sequential presentations of different odors. The odor sequence used was water (reference), vanilla (non-social cue) and urine from males and females (social odors) embedded in a $2 \times 2 \mathrm{~cm}$ piece of cotton. Each odor (or water) was presented in three consecutive trials for a duration of $2 \mathrm{~min}$. The inter-trial interval was $1 \mathrm{~min}$. The sniffing time of the cotton piece (nose tip less than $1 \mathrm{~cm}$ from the cotton) was quantified.

\section{Intruder Test}

The protocol was adapted from previously described methods (Gascon et al., 2014). To avoid any aggressive behaviors, a juvenile CD1 male (3-5 weeks) was introduced into the home cage of the test mouse and the interactions were recorded for $5 \mathrm{~min}$. The time and the number of interactive and investigative behaviors (sniffing) initiated by the target mouse were quantified.

\section{Interactions in Neutral Arena}

In this task, the target mouse and a juvenile CD1 male (3-5 weeks) were simultaneously introduced into an open field arena (see before). The time and the number of interactive and investigative behaviors (sniffing) initiated by the target mouse during the $5 \mathrm{~min}$ of the test were assessed.

\section{Three Chamber Social Task}

The three chamber apparatus is a rectangular arena $(60 \times 37.5 \times 21 \mathrm{~cm})$ made of transparent plexiglass plastic, divided into three compartments of the same size $(18.5 \times 37 \times 21 \mathrm{~cm})$. Two openings connect the center chamber with the two side chambers. In a corner of each side chamber, there is a cylindrical container $(10 \mathrm{~cm}$ in diameter, $20 \mathrm{~cm}$ high). During the first trial (exploration), the target mouse was placed in the middle chamber facing a wall and allowed to explore. In the next trial (social), a stranger mouse was placed in one of the containers while the other was empty. In the last trial (memory), the same mouse was placed on one side but a novel stranger was placed in the other container. Trials lasted $10 \mathrm{~min}$ and animals were left for $10 \mathrm{~min}$ in their home cages between trials. Strangers were juvenile CD1 male mice (3-5 weeks) that had been trained to be restrained in the container. For each mouse, the location of the stranger mice in the left or right side chamber was random. For the analysis, the stranger in the social phase was arbitrarily assigned to the left chamber and the novel stranger in the social trial was placed to the right chamber. A left-right index (LRI) was calculated as the subtraction of the close exploration time (nose point within $2 \mathrm{~cm}$ of the container) from the left and right containers. To quantify the behavior, only the first $5 \mathrm{~min}$ of each trial were used.

\section{Device Cleaning}

Between trials or animals, behavioral devices were thoroughly cleaned with water and mild soap. After rinsing, surfaces were sprayed with a $70 \%$ ethanol solution and air-dried for $5 \mathrm{~min}$.

\section{Data Analysis and Statistics}

For tests involving two freely moving animals (intruder test and interactions in the neutral arena), an investigator blind to the conditions manually scored the behaviors. For the other tests, mice tracking and behavioral analysis were carried out using dedicated software (Ethovision XT, Noldus, Netherlands). Data are presented as means \pm SEM unless indicated. Statistical analyses, either nonparametric two-sample Kolmogorov-Smirnov test to compare the distribution of 3- and 9-month-old mice, or analysis of variance (ANOVA) for repeated measures followed by Sidak's post hoc multiple comparison test, were performed using Prism GraphPad Software (version 7). The significance was set at $p<0.05$ for all statistical tests.

\section{RESULTS}

\section{Middle-Aged Mice Show No Gross Deficit in Basal Activity}

Before submitting young (3-month-old) and middle-aged (9month-old) mice to further behavioral phenotyping, we sought to rule out the possibility that gross behavioral abnormalities existed in these animals, either at the cohort level or at the individual level. For that purpose, we monitored both groups in their home cages over a 20 -h period (PhenoTyper). We found that, during this period, there were no differences between both groups of animals in most of the parameters evaluated (Figure 1). Thus, young and middle-aged animals have a similar activity profile, including feeding, water consumption and resting in the shelter area. For these activities, neither the frequency (2-way ANOVA for repeated measures, Factor age, $F_{(1,45)}=0.02, p=0.887$ ) nor the time spent (2-way ANOVA for repeated measures, Factor age, $\left.F_{(1,45)}=0.81, p=0.372\right)$ showed significant differences between the animals at 3 and 9 months. Additionally, food and water intake were also indistinguishable between the two groups (2-way ANOVA for repeated measures, Factor age, $F_{(1,45)}=0.05$, $p=0.820)$, suggesting that in terms of basal activity, middle-aged mice did not exhibit any impairment that would preclude the assessment of brain functions in terms of behavior.

\section{Middle-Aged Mice Show a Decrease in Locomotion but Normal Exploration}

It has been widely reported that locomotion decreases with age in several species. We, therefore, quantified the distance 

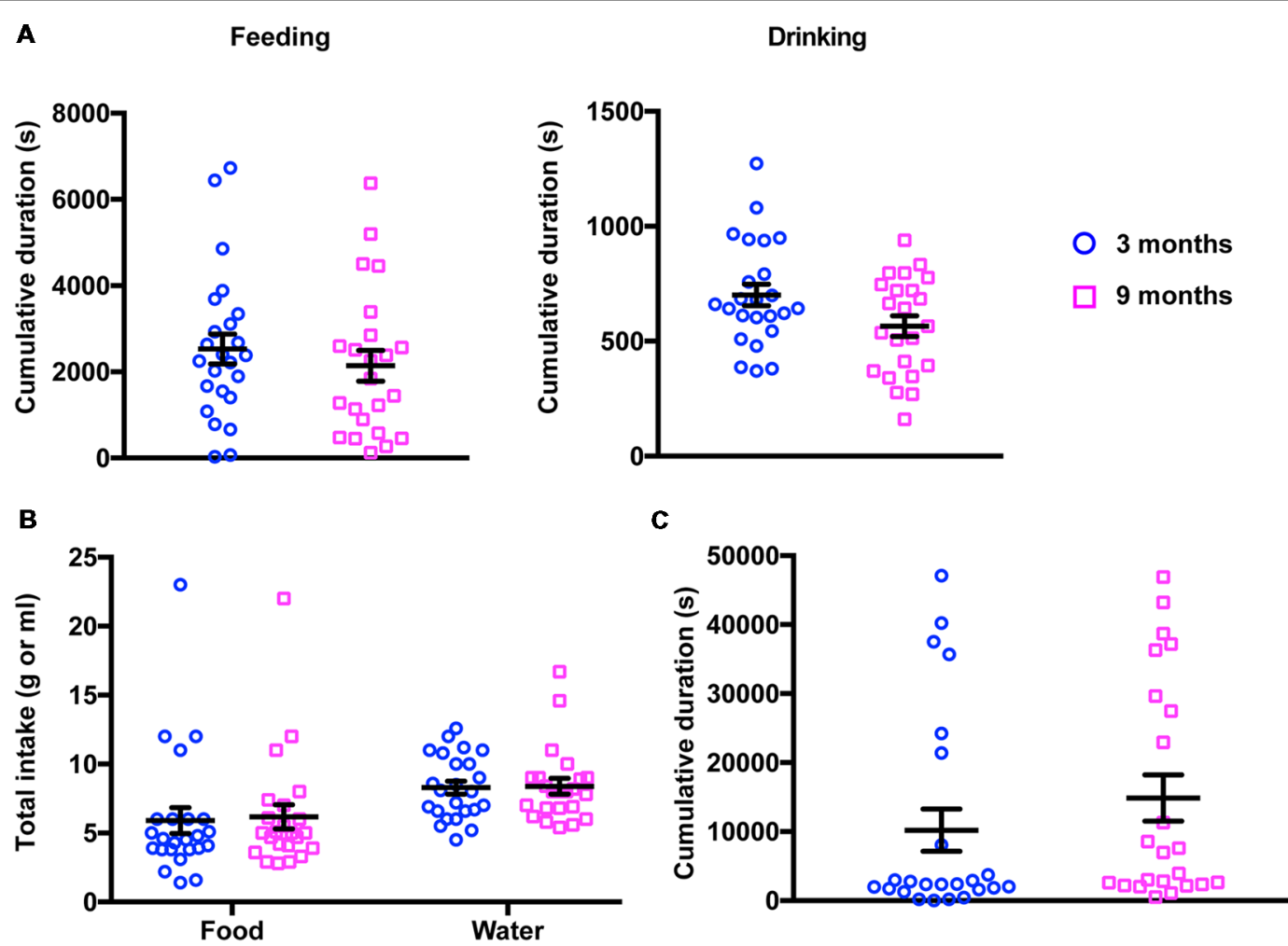

FIGURE 1 | Results of 20-h activity monitoring in the PhenoTyper. (A) No differences were detected in the total time spent feeding (left panel) and drinking (right panel) between 3- and 9-month-old mice (3 months, feeding 2,530 $\pm 349.7 \mathrm{~s}$, drinking $701.1 \pm 46.49 \mathrm{~s} ; 9$ months, feeding 2,142 $\pm 356.0 \mathrm{~s}$, drinking $565.8 \pm 44.60 \mathrm{~s}$ ). (B) Young and middle-aged mice showed a similar food and water consumption (3 months, food $5.91 \pm 0.94 \mathrm{~g}$, water $8.29 \pm 0.47 \mathrm{ml} ; 9 \mathrm{months}$, food $6.18 \pm 0.87 \mathrm{~g}$, water $8.39 \pm 0.57 \mathrm{ml})$. (C) The cumulative time spent in the shelter did not change in 3 - vs. 9-month-old mice ( 3 months, 10,210 $\pm 3,070 \mathrm{~s}$; 9 months, $14,870 \pm 3,359$ s).

traveled by the animals during the observation period in the PhenoTyper (Figure 2A, left panel). We found that 9-monthold mice moved significantly less than younger mice (total distance, 3 months $76.48 \pm 3.54 \mathrm{~m}$; 9 months $58.84 \pm 3.05 \mathrm{~m}$, mean \pm SEM, $p=0.0026$, Kolmogorov-Smirnov test). We confirmed these findings in the open field test where animals were allowed to explore an unknown arena for only $10 \mathrm{~min}$ (Figure 2A, right panel). A similar reduction in the total distance traveled by animals was found in middle-aged mice (total distance, 3 months $3.43 \pm 1.86 \mathrm{~m}$; 9 months $2.77 \pm 1.83 \mathrm{~m}$, mean \pm SEM, $p=0.0219$, KolmogorovSmirnov test). Interestingly, the exploratory behavior of mice (the time spent near walls vs. time spent in the center of the arena) remained undistinguishable in both groups (Figure 2B) suggesting that, even if locomotion is reduced, this does not lead to a change in basic exploratory behavior.

\section{Middle-Aged Mice Show No Alteration in Cognitive Tasks}

We next examined cognitive functions using two well-established independent paradigms, the novel object recognition and the MWM. In the novel object recognition task, mice were tested for $10 \mathrm{~min}$ in an open field arena containing two identical (exploration phase) or different (recognition phase) objects placed in the opposite wall of the release zone. To quantify the exploratory behavior of mice, including object recognition and memory, a RI was calculated (see "Materials and Methods" section). Figure 3A summarizes the performances of 3-monthold and 9-month-old animals in this test. As expected, the young mice examined both objects similarly during the exploration phase, but showed a clear preference for the novel object during the recognition phase $\left(\mathrm{RI}_{\text {exploration }}=0.50 \pm 0.04\right.$; $\mathrm{RI}_{\text {recognition }}=0.65 \pm 0.04 ; 2$-way ANOVA for repeated measures, Factor phase, $F_{(1,44)}=14.9, p=0.0004$, adjusted $p$-value for multiple comparisons: $p=0.0252)$. Middle-aged mice exhibited similar behavior $\left(\mathrm{RI}_{\text {exploration }}=0.50 \pm 0.05\right.$; $\mathrm{RI}_{\text {exploration }}=0.65 \pm 0.03$, 2-way ANOVA for repeated measures, Factor phase, $F_{(1,44)}=14.9, p=0.0004$, adjusted $p$-value for multiple comparisons: $p=0.0129$ ). No significant differences could be observed between the two groups of mice (2-way ANOVA for repeated measures, Factor age, $F_{(1,44)}=1.04 \mathrm{E}-4$, $p=0.992$ ) suggesting that moderate aging had little impact on the functions required for this task.

To further support our findings, both mouse cohorts were submitted to the MWM, a spatial memory and learning assessment task. As shown in Figure 3B (left panel), during the training phase, escape latency gradually decreased over the training days in both groups, suggesting that young and old mice 

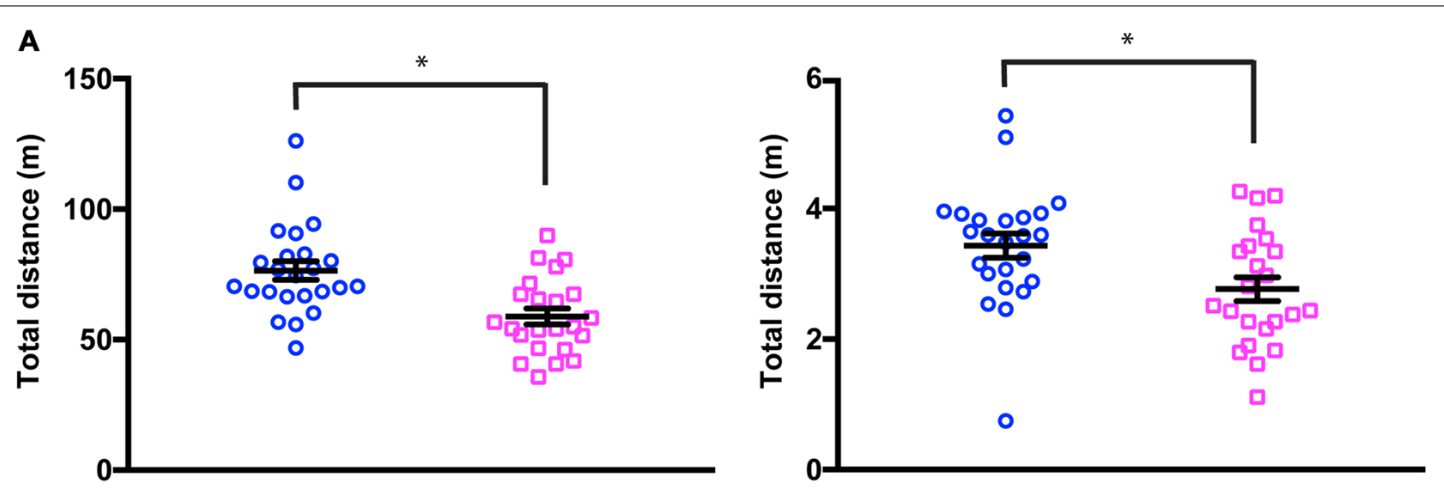

B

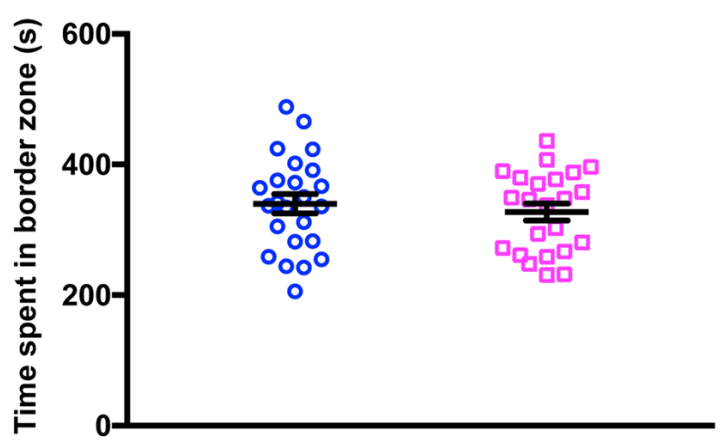

3 months

9 months

FIGURE 2 | Locomotion is reduced in 9-month-old mice. (A) Total distance traveled in the PhenoTyper (20-h period, left panel) and in the open field (10-min period, right panel) was significantly decreased in 9-month-old mice (3 months, Phenotyper $76.48 \pm 3.54 \mathrm{~m}$, open field $3.43 \pm 1.86 \mathrm{~m}$; 9 months, PhenoTyper $58.84 \pm 3.04 \mathrm{~m}$, open field $2.77 \pm 1.83 \mathrm{~m}$ ). PhenoTyper ${ }^{*} p=0.0026$, open field ${ }^{*} p=0.0219$ (Kolmogorov-Smirnov test). (B) Time spent in close proximity to the walls of the open field was not modified in 3- vs. 9-month-old mice (3 months, $339.9 \pm 14.82 \mathrm{~s} ; 9$ months, $327.35 \pm 12.90 \mathrm{~s}$ ).

learned to use spatial information to navigate to the submerged platform (2-way ANOVA, Factor training day, $F_{(3,14)}=6.92$, $p=0.0002$, adjusted $p$-value for multiple comparisons day 1 vs. day $4: p_{3}$ months $=0.036$ and $p_{9}$ months $=0.006$ ). Importantly, the learning curves of young and middle-aged mice were identical (2-way ANOVA for repeated measures, Factor age, $F_{(1,45)}=1.22, p=0.25$ ). Similarly, in the probe trial (Figure 3B, right panel), middle-aged animals had the same latency times and platform crossovers as young mice (2-way ANOVA, Factor age, $F_{(1,90)}=0.231, p=0.632$, adjusted $p$-value for multiple comparisons: $p_{\text {frequency }}=0.968$, $\left.p_{\text {latency }}=0.600\right)$, indicating again no overt impairment in cognitive functions.

\section{Middle-Aged Mice Show No Impairment in Olfactory Function}

Since mouse behavior largely relies on olfactory cues, we then examined olfactory functions in young and middle-aged mice. To do this, both groups of animals were exposed sequentially to water (baseline investigation), vanilla (as an attractive odorant) and urine from two different mice (as social-related cues). Our analysis revealed that both groups of animals display essentially the same olfactory exploration pattern including an increase in investigation time upon exposure to a novel odor and dishabituation in subsequent trials (Figure 3C). In addition, social cues (male and female urine) elicited much stronger responses than vanilla (Figure $3 \mathrm{C}$ ). No difference could be found at any trial between 3-month-old and 9-month-old mice (2-way ANOVA, Factor age, $\left.F_{(1,494)}=9.09 \mathrm{E}-5, p=0.992\right)$ indicating that aging does not disrupt olfactory discrimination or olfactory sensitivity.

\section{Social Behavior Is Altered in Middle-Aged Mice}

We next sought to investigate whether or not the social functions of middle-aged mice were affected. In order to obtain a comprehensive description of social behaviors, we conducted three independent paradigms, the 3-chamber task, the interactions in the neutral arena and the intruder test.

We first carried out the 3-chamber paradigm divided into three trials (see "Materials and Methods" section). By measuring the time spent by animals investigating empty objects or the different social partners in the side compartments (LRI, see "Materials and Methods" for the details), these different trials enabled assessment of exploratory behavior, social recognition and social memory, respectively. As shown in Figure 4A, both young and middle-aged mice displayed the expected behavioral pattern; LRI was close to 0 during the exploration phase $\left(\mathrm{LRI}_{3}\right.$ months $=16.03 \pm 8.28 ; \mathrm{LRI}_{9}$ months $\left.=9.00 \pm 7.96\right)$, whereas there was a clear preference for the left chamber during the social trial $\left(\mathrm{LRI}_{3}\right.$ months $=43.36 \pm 5.47 ; \mathrm{LRI}_{9}$ months $\left.=29.76 \pm 5.06\right)$ 


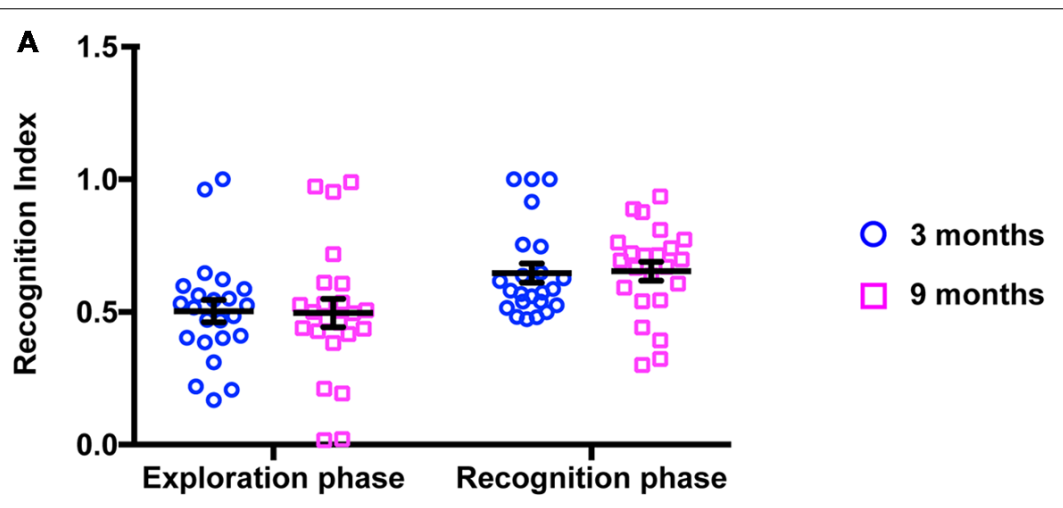

$\mathbf{B}$
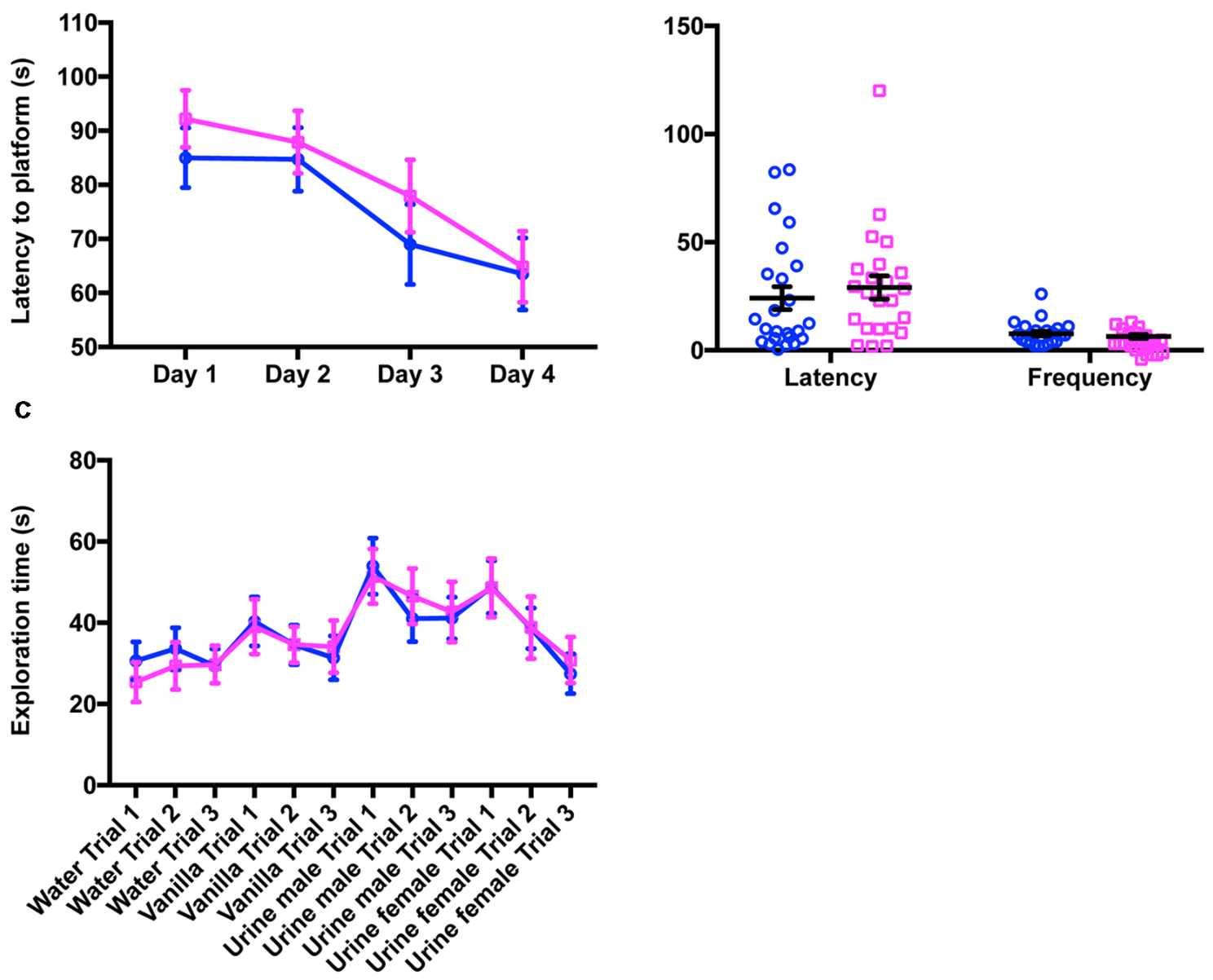

FIGURE 3 | Cognitive function and olfaction are not impaired in 9-month-old mice. (A) In the novel object recognition task (10-min period), the proportion of time spent exploring the objects recognition index (RI) did not change in 9-month-old animals, neither during the exploration phase (two identical objects) nor during the recognition phase (one familiar object, one novel object; 3 months, $0.50 \pm 0.04$; R $1_{\text {recognition }}=0.65 \pm 0.04 ; 9$ months, Rl exploration $=0.50 \pm 0.05$;

$\mathrm{Rl}_{\text {exploration }}=0.65 \pm 0.03$ ). (B) Quantification of the performances of 3- and 9-month-old mice in MWM did not reveal any difference in latency to reach the platform during training (left panel; 3 months, Day $185.00 \pm 5.53$ s, Day $284.70 \pm 5.88$ s, Day $368.98 \pm 7.39$ s, Day $463.51 \pm 6.66$ s; 9 months, Day $192.18 \pm 5.28$ s, Day $287.90 \pm 5.77 \mathrm{~s}$, Day $377.93 \pm 6.71 \mathrm{~s}$, Day $464.85 \pm 6.57 \mathrm{~s}$ ) or during the probe session (right panel; 3 months, Latency $24.13 \pm 5.28 \mathrm{~s}$, Frequency $7.71 \pm 1.08 ; 9$ months, Latency $29.06 \pm 5.42 \mathrm{~s}$, Frequency $6.48 \pm 0.89$ ). (C) In the habituation/dishabituation olfactory task, 3- and 9-month-old mice were indistinguishable and spent equivalent amount of time investigating water, vanilla and urine from males or females ( 3 months, Water Trial $130.63 \pm 4.69 \mathrm{~s}$, Water Trial 233.65 土 5.14 s, Water Trial $329.34 \pm 4.24$ s; 3 months, Vanilla Trial $140.32 \pm 6.02$ s, Vanilla Trial $234.56 \pm 4.85$ s, Vanilla Trial $331.36 \pm 5.37$ s; Urine Male Trial $153.94 \pm 6.89 \mathrm{~s}$, Urine male Trial $241.00 \pm 5.61 \mathrm{~s}$, Urine male Trial $341.17 \pm 5.11 \mathrm{~s}$, Urine female Trial $148.82 \pm 6.49 \mathrm{~s}$, Urine female Trial $238.64 \pm 4.98 \mathrm{~s}$, Urine female Trial $327.46 \pm 4.91$ s; 9 months, Water Trial $125.41 \pm 4.89 \mathrm{~s}$, Water Trial $229.38 \pm 5.82 \mathrm{~s}$, Water Trial $329.73 \pm 4.66 \mathrm{~s}$, Vanilla Trial $139.03 \pm 6.74 \mathrm{~s}$, Vanilla Trial $234.61 \pm 4.41 \mathrm{~s}$, Vanilla Trial $334.12 \pm 6.45 \mathrm{~s}$, Urine male Trial $151.42 \pm 6.72 \mathrm{~s}$, Urine male Trial $246.56 \pm 6.82 \mathrm{~s}$, Urine male Trial $342.67 \pm 7.42 \mathrm{~s}$, Urine female Trial $148.60 \pm 7.22 \mathrm{~s}$, Urine female Trial $238.81 \pm 7.62 \mathrm{~s}$, Urine female Trial $330.84 \pm 5.69 \mathrm{~s})$. 
and a shift to the right side was observed in the last trial $\left(\mathrm{LRI}_{3}\right.$ months $=-12.67 \pm 2.26 ; \mathrm{LRI}_{9}$ months $\left.=-17.13 \pm 6.40\right)$. Statistical analysis of these data confirmed that there were no significant differences at any trial between 3- and 9-month-old mice (2-way ANOVA for repeated measures, Factor age, $F_{(1,45)}=2.40, p=0.129$, adjusted $p$-value for multiple comparisons: $p_{\text {exploration }}=0.811, p_{\text {social }}=0.329$, $\left.p_{\text {memory }}=0.942\right)$. Other parameters usually quantified in these tests such as the number of bouts or the time spent in each chamber were not modified either (data not shown) suggesting that fundamental social functions such as recognition of a conspecific and memory remained intact in middle-aged animals.

We next measured the social interactions of our two groups of animals in a neutral arena where they were exposed to an unfamiliar juvenile male mouse (3-5 weeks old) for $5 \mathrm{~min}$. In this task, mice were confronted with a new physical and social environment and their social behavior is quantified by the time as well as the number/nature of interactions initiated by the target mouse (Figure 4B, left panel). Although young mice spent more time in social interactions than middle-aged mice (Figure 4B, central panel, $56.46 \pm 4.84$; vs. $41.61 \pm 3.36$, mean \pm sem, $p=0.0196$, two-tails KolmogorovSmirnov test), the number of different interactions remained unchanged (2-way ANOVA for repeated measures, Factor age, $F_{(1,45)}=3.84, p=0.0563$, adjusted $p$-value for multiple comparisons: $\left.p_{\text {nose }}=0.999, p_{\text {ano-genital }}=0.0613, p_{\text {other }}=0.350\right)$. We further investigated this decrease in social interest by analyzing the interactions at $1 \mathrm{~min}$ intervals (Figure $\mathbf{4 B}$, right panel). We observed that, among young animals, exploration of the social partner peaked at the 1 st $\mathrm{min}$ and remained at lower levels thereafter. In contrast, 9-month-old mice spent much less time in social contact at the beginning of the test (2-way ANOVA for repeated measures, Factor age, $F_{(1,45)}=7.09, p=0.0161$, adjusted $p$-value for multiple comparisons: $p_{\text {minute } 1}=0.009, p_{\text {minute } 2}=0.736$, $p_{\text {minute } 3}=0.763$, $\left.p_{\text {minute } 4}=0.895, p_{\text {minute }} 5=0.906\right)$. These observations suggest that social exploratory behavior, particularly social interest, is altered in middle-aged mice.

To confirm these findings, we further assessed social functions in the intruder test. In this task, a stranger male juvenile mouse (3-5 weeks-old) was introduced into the home cage, thus avoiding the need for simultaneous investigation of a novel arena. In this test, time and the number/nature of interactions were also used as an output of the social function. Figure $4 \mathrm{C}$ summarizes the results of this test; we found that the number of each kind of interaction was not different between young and middle-aged mice (Figure 4C, left panel, 2-way ANOVA for repeated measures, Factor age, $F_{(1,45)}=2.45, p=0.125$, adjusted $p$-value for multiple comparisons: $p_{\text {nose }}=0.999, p_{\text {ano-genital }}=0.550$, $\left.p_{\text {other }}=0.136\right)$. However, consistent with our findings in the neutral arena, the total time spent in social interactions was significantly reduced (Figure 4C, middle panel, 3 months $123.8 \pm 11.72$ for young mice vs. $83.91 \pm 6.84$ for older mice, $p=0.0496$, two-tails Kolmogorov-Smirnov test). When the interaction time was analyzed in 1-min splitting (Figure 4C, right panel), we observed that young animals explored the intruder in depth during the first $2 \mathrm{~min}$ of the test and that the interactions shortened subsequently. In the 9-monthold mice, social contacts were maintained at similar levels for the 1st min, but dropped significantly for the remainder of the test duration (2-way ANOVA for repeated measures, Factor age, $F_{(1,45)}=7.09, p=0.0106$, adjusted $p$-value for multiple comparisons: $p_{\text {minute } 1}=0.998, p_{\text {minute } 2}=0.006$, $p_{\text {minute } 3}=0.0864, p_{\text {minute } 4}=0.467, p_{\text {minute } 5}=0.316$ ) arguing for an age-dependent reduction in social drive already detectable in 9-month-old mice.

\section{DISCUSSION}

In this study, we characterized young and middle-aged mice in social and non-social tasks. Behavioral test batteries have been used to assess a wide variety of behavioral traits. Our main findings for middle-aged mice are as follows: (i) their locomotion is significantly reduced; (ii) they have no overt impairment in multiple brain functions, including exploration, olfaction, learning and spatial and working memory; and (iii) they exhibit altered social behavior, specifically in terms of social drive/sociability.

Motor activity in the open field is known to decrease in aging mice and rats (Lalonde and Badescu, 1995; Lalonde and Strazielle, 2009). Previous work (Fahlström et al., 2011) has suggested that locomotion decline in middle-aged mice is exclusively associated with exploratory tasks, even when animals have been analyzed over longer time periods. Conversely, we found that $\mathrm{C} 57 \mathrm{Bl} / \mathrm{J}$ males exhibit a reduction in the distance traveled not only in the open field (explorationrelated locomotion) but also in the context of their home cage (basal locomotion). Our findings therefore argue for a gradual loss of locomotor activity with age, in accordance with an abundant literature reporting such a decline in a striking number of species, from C. elegans to rodents and primates (Ingram, 1988, 2000; Walton et al., 2006; Fahlström et al., 2012; Marck et al., 2017).

Many studies have addressed the impact of aging in rodents' cognitive functions using different behavioral paradigms (Wood and Dudchenko, 2003; LaSarge et al., 2007; Bizon et al., 2012). In mice, novel object recognition and MWM have been widely used. In the former test, middle-aged mice (9-12 months) show similar performance levels to their younger counterparts (Benice et al., 2006; Davis et al., 2012; Fahlström et al., 2012). Similarly, most studies failed to detect differences in MWM until 16-18 months (Calhoun et al., 1998; Frick et al., 2002; Benice et al., 2006; de Fiebre et al., 2006; Murphy et al., 2006; Davis et al., 2012; Fahlström et al., 2012). Our findings using 9-month-old mice are consistent with these observations. More importantly, evidence suggesting that cognitive functions remain largely intact in middle-aged mice have been obtained using additional behavioral tasks (i.e., Barnes maze or radial maze; Barreto et al., 2010; Pettan-Brewer et al., 2013). Collectively, these data indicate that 9-month-old mice used in this work are tested well before the onset of any cognitive decline. 
A

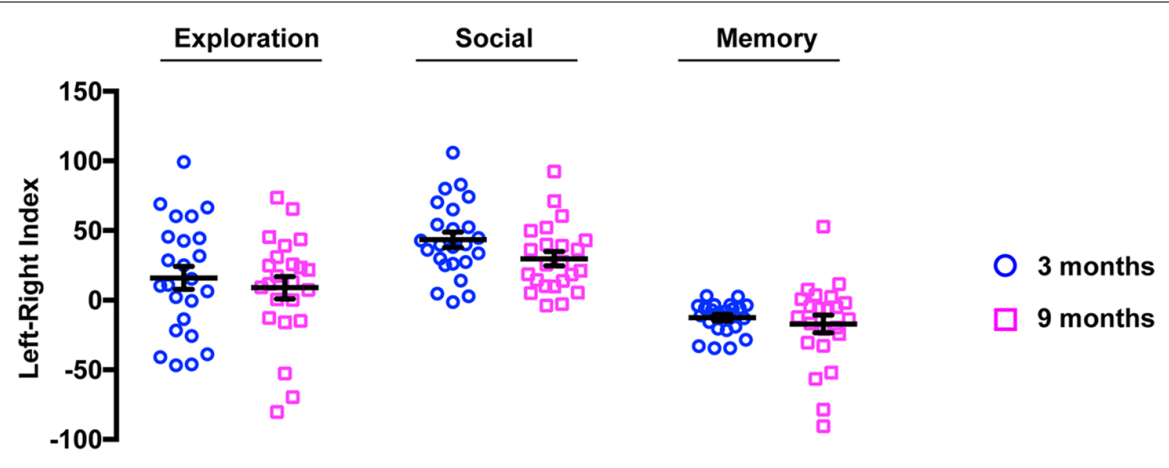

B
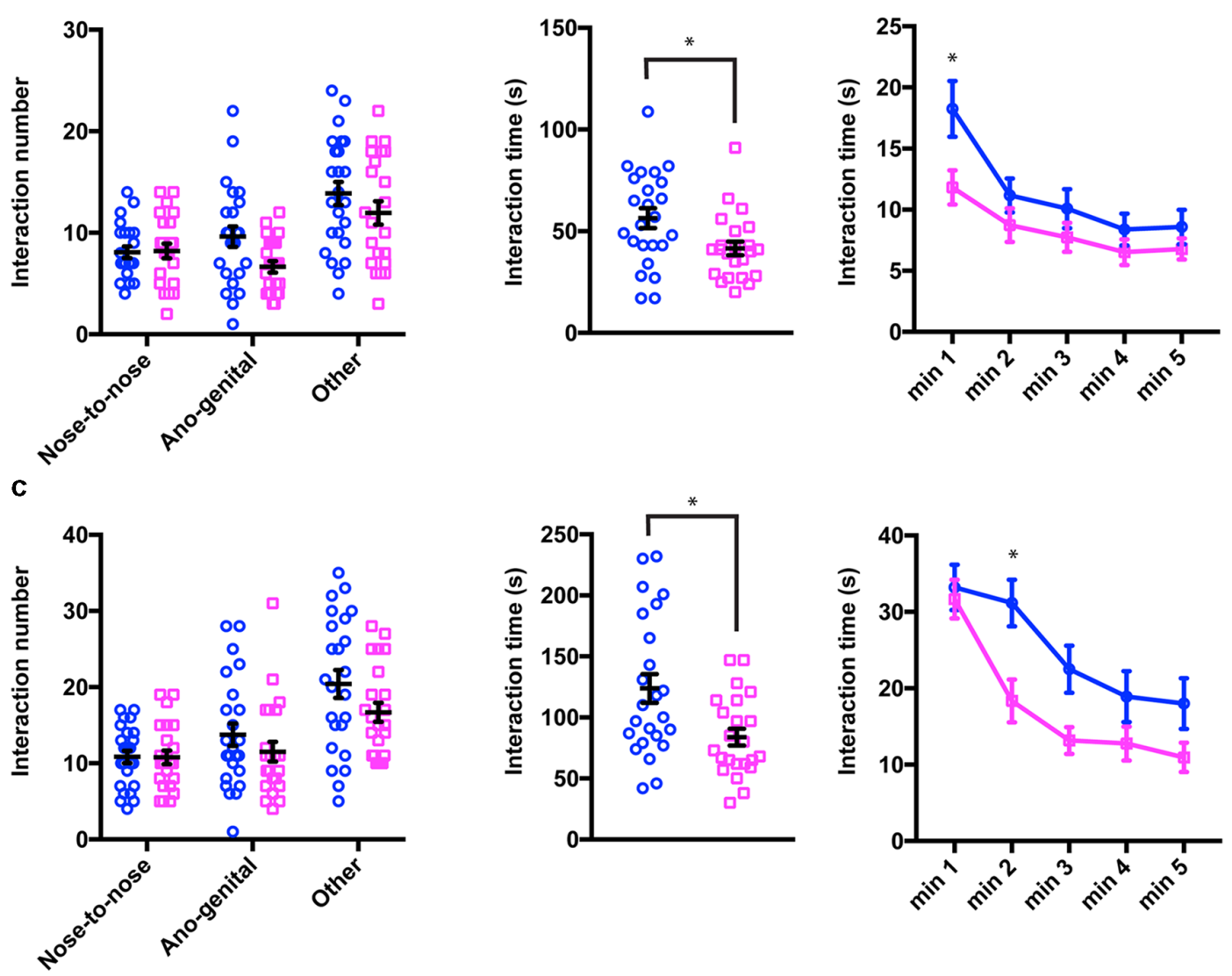

FIGURE 4 | Social drive/sociability is specifically disrupted in 9-month-old mice. (A) In the three-chamber apparatus, the mice spent the same amount of time exploring empty objects (exploration trial) or the social partners (social and memory phases) regardless of their age. For quantification, a left-right index (LRI) was calculated (see "Materials and Methods" section; 3 months, LRI $\left.\right|_{\text {exploration }} 16.03 \pm 8.28,\left.L R\right|_{\text {exploration }} 29.76 \pm 5.06, L_{\text {social }}-12.67 \pm 2.26$; 9 months, LRI $I_{\text {social }}$ $9.00 \pm 7.96, L R I_{\text {memory }} 43.36 \pm 5.47, \mathrm{LRI}_{\text {memory }}-17.13 \pm 6.40$ ). (B) Quantification of social interactions initiated by 3 - and 9-month-old mice in a novel arena containing a juvenile male ( $3-5$ week-old). Although the number and type of interactions (left panel; 3 months, Nose-to-nose $8.08 \pm 0.56$, Ano-genital 9.62 \pm 1.02 , Other, $13.87 \pm 1.14 ; 9$ months, Nose-to-nose $8.22 \pm 0.72$, Ano-genital $6.65 \pm 0.56$, Other, $11.96 \pm 1.14$ ) showed no difference between the two groups, the total time spent interacting was significantly lower in elderly mice (middle panel; 3 months, $56.46 \pm 4.84 \mathrm{~s} ; 9$ months, $41.61 \pm 3.36 \mathrm{~s}$, ${ }^{*} p=0.0196$, Kolmogorov-Smirnov test). When the interaction time was analyzed at 1 -min intervals (right panel), most of the reduction was observed in the 1 st min of the exploration phase (3 months, Minute $118.25 \pm 2.28 \mathrm{~s}$, Minute $211.16 \pm 1.38 \mathrm{~s}$, Minute $310.08 \pm 1.60 \mathrm{~s}$, Minute $48.37 \pm 1.31 \mathrm{~s}$, Minute $58.58 \pm 1.40 \mathrm{~s} ; 9$ months, Minute $111.82 \pm 1.39 \mathrm{~s}$, Minute $28.74 \pm 1.37 \mathrm{~s}$, Minute $37.74 \pm 1.19 \mathrm{~s}$, Minute $46.52 \pm 1.05 \mathrm{~s}$, Minute $56.78 \pm 0.86 \mathrm{~s}$ ). ${ }^{*}{ }^{*}$ minute $1=0.009$ [2 way analysis of variance (ANOVA), Sidak test]. (C) In the intruder test, the number and type of interactions (left panel; 3 months, Nose-to-nose 10.83 \pm 0.83 , Ano-genital 13.75 \pm 1.48 , Other, $20.42 \pm 1.82$; 9 months, Nose-to-nose $10.78 \pm 0.91$, Ano-genital $11.52 \pm 1.30$, Other, $16.70 \pm 1.25$ ) were not altered in 9-month-old mice compared to 3-month-old animals. In contrast, the total time spent interacting was significantly lower in older mice (middle panel; 3 months, $123.8 \pm 11.72 \mathrm{~s} ; 9$ months, $83.91 \pm 6.84 \mathrm{~s},{ }^{*} p=0.0496$, Kolmogorov-Smirnov test). At 1 min intervals (right panel), the 9-month-old mice showed no change in the exploration phase (first minute), but a significant decrease in social afterwards (3 months, Minute $133.20 \pm 2.97 \mathrm{~s}$, Minute $231.17 \pm 3.04 \mathrm{~s}$, Minute $322.50 \pm 3.07 \mathrm{~s}$, Minute $418.92 \pm 3.43 \mathrm{~s}$, Minute $518.00 \pm 3.30 \mathrm{~s}$; 9 months, Minute $131.70 \pm 2.53 \mathrm{~s}$, Minute $218.34 \pm 2.79 \mathrm{~s}$, Minute $313.17 \pm 1.678 \mathrm{~s}$, Minute $412.78 \pm 2.22 \mathrm{~s}$, Minute $510.96 \pm 1.92 \mathrm{~s}) .{ }^{*} p_{\text {minute2 }}=0.006$ (2-way ANOVA, Sidak test). 
Although aging seems to spare sensory functions (Fahlström et al., 2011), decreased olfactory function is very common (>50\%) in humans over 65 (Attems et al., 2015). Old mice (from 18 to 24 months) also show impairments in olfactory discrimination and memory (Enwere et al., 2004; Patel and Larson, 2009; Moreno et al., 2014). Given the relevance of olfactory cues to the social behavior of mice (Dulac and Torello, 2003; Dulac, 2006), it was essential to rule out that social alterations in middle-aged mice were not due to olfactory malfunction. We observed no differences between our two groups of mice in olfactory tasks, indicating that moderate aging may not cause olfactory deficits. Moreover, a recent report reveals that the discrimination abilities of middle-aged (10 months) mice may be even more refined than those of young (2 months) or old (23 months) animals (Rey et al., 2012), which also argues against this possibility.

The major finding in this report is the existence of social impairments in middle-aged mice. By assessing social behavior according to three independent paradigms, we were able to obtain a detailed analysis of social functions and uncover relatively subtle changes. The absence of differences in the threechamber test suggests that middle-aged mice maintain their basic social abilities, including social exploration, recognition of a conspecific, social interest and memory. The results of the neutral arena indicate an overall decrease in the time spent on social interactions, especially during the exploratory phase (1st min of the test). A recent study reported similar results in this test in mice aged 8-12 months (Shoji et al., 2016). In this task, the mice simultaneously investigate a new environment and a social partner complicating the interpretation of the results. However, we did not observe any difference in the exploratory behavior in middle-aged mice either in the open field or in the novel object recognition (data not shown) arguing against the possibility that neutral arena deficits arise from alterations in exploratory functions. More importantly, middle-aged mice exhibited similar defects in the intruder test. Although mainly used to evaluate aggression, this test could also be used to evaluate sociability when the intruder is a non-aggressive partner (either a female or a juvenile; Moles et al., 2007; Ey et al., 2012). Here, we favor a juvenile male as a social target to avoid the confounding effect of mating in male-female dyads. We show that, even in the context of their home cage, middle-aged mice exhibit a similar reduction in interaction time compared to young animals. Interestingly, a more detailed analysis revealed that, during the 1st min of the task (active exploration of the intruder), there is no difference in the interaction time between young and middle-aged mice suggesting that social exploration and social interest are not perturbed. Then, 3-month-old animals maintain equally high levels of social interactions during the second minute whereas, in 9-month-old mice, they have dropped significantly and remain lower for the rest of the test. These findings suggest that aging may initially disrupt social drive/sociability. Remarkably, similar deficits have been described in different mouse models of frontotemporal dementia, an early onset neurodegenerative disease primarily affecting social behavior (Filiano et al., 2013; Gascon et al., 2014; Chew et al., 2015).
It will be important in the future to narrow the window of time when social impairment begins. Nevertheless, it can be difficult to reconcile the exact same findings of an age-related decline in different mouse strains due to robust strain differences. For example, some mouse strains do not learn the spatial MWM task slowly, whereas other strains learn the task very easily, thus confounding the observation of age-related changes in performance (Upchurch and Wehner, 1988a,b). Therefore, more than defining a moment, it will be crucial investigating the brain areas involved in early decline of social activities using objective neural measurements such as functional magnetic resonance imaging and optogenetics (Benekareddy et al., 2018).

As a major limitation of our work, we have not addressed the differences between male and female in the different behavioral traits observed. Indeed, changes in behavior with age could be quantitatively different between males and females but could also vary qualitatively (in terms of signification). For example, spatial reference memory decline begins at an earlier age in females than in males, a finding that may be related to the cessation of estrous cycling (Frick et al., 2000). It has been shown that the spontaneous failure of the estrous cycle increased anxiety, suggesting that the perimenopausal period has a significant influence on anxiety-related behaviors in female (Guimarães et al., 2015). The use of factorial analyses would make it possible to identify the motivational drivers underlying the spontaneous behavior of both sexes at different periods in their lives and to identify possible relationships between the indices of the different tests. Future analyses should focus on sexual differences in behaviors expressed in conventional social tests.

Collectively, these findings open the intriguing possibility that neuronal circuits underlying specific domains of social functions (such as social drive/sociability) may be particularly susceptible to the aging process and may drive early symptoms of pathological aging.

\section{DATA AVAILABILITY}

The datasets generated for this study are available on request to the corresponding author.

\section{AUTHOR CONTRIBUTIONS}

FB performed the behavioral tests. FB, FJ and EG analyzed the data. EI and EG wrote the manuscript.

\section{FUNDING}

This study was supported by grants from The French Research Agency (Agence Nationale de la Recherche; ANR15-CE16-0008-01) to EG.

\section{ACKNOWLEDGMENTS}

We thank Ana Borges-Correia, Elodie Caccomo-Garcia, Catherine Lepolard and Natalia Popa for technical assistance. 


\section{REFERENCES}

Almeling, L., Hammerschmidt, K., Sennhenn-Reulen, H., Freund, A. M., and Fischer, J. (2016). Motivational shifts in aging monkeys and the origins of social selectivity. Curr. Biol. 26, 1744-1749. doi: 10.1016/j.cub.2016.04.066

Almeling, L., Sennhenn-Reulen, H., Hammerschmidt, K., Freund, A. M., and Fischer, J. (2017). Social interactions and activity patterns of old Barbary macaques: further insights into the foundations of social selectivity. Am. J. Primatol. 79:e22711. doi: 10.1002/ajp.22711

Attems, J., Walker, L., and Jellinger, K. A. (2015). Olfaction and aging: a mini-review. Gerontology 61, 485-490. doi: 10.1159/000381619

Barreto, G., Huang, T. T., and Giffard, R. G. (2010). Age-related defects in sensorimotor activity, spatial learning and memory in C57BL/6 mice. J. Neurosurg. Anesthesiol. 22, 214-219. doi: 10.1097/ana.0b013e3181d $56 c 98$

Benekareddy, M., Stachniak, T. J., Bruns, A., Knoflach, F., von Kienlin, M., Künnecke, B., et al. (2018). Identification of a corticohabenular circuit regulating socially directed behavior. Biol. Psychiatry 83, 607-617. doi: 10.1016/j.biopsych.2017.10.032

Benice, T. S., Rizk, A., Kohama, S., Pfankuch, T., and Raber, J. (2006). Sexdifferences in age-related cognitive decline in C57BL/6J mice associated with increased brain microtubule-associated protein 2 and synaptophysin immunoreactivity. Neuroscience 137, 413-423. doi: 10.1016/j.neuroscience. 2005.08.029

Bishop, N. A., Lu, T., and Yankner, B. A. (2010). Neural mechanisms of ageing and cognitive decline. Nature 464, 529-535. doi: 10.1038/nature08983

Bizon, J. L., Foster, T. C., Alexander, G. E., and Glisky, E. L. (2012). Characterizing cognitive aging of working memory and executive function in animal models. Front. Aging Neurosci. 4:19. doi: 10.3389/fnagi.2012.00019

Bottiroli, S., Cavallini, E., Ceccato, I., Vecchi, T., and Lecce, S. (2016). Theory of mind in aging: comparing cognitive and affective components in the faux pas test. Arch. Gerontol. Geriatr. 62, 152-162. doi: 10.1016/j.archger.2015. 09.009

Calhoun, M. E., Kurth, D., Phinney, A. L., Long, J. M., Hengemihle, J., Mouton, P. R., et al. (1998). Hippocampal neuron and synaptophysin-positive bouton number in aging C57BL/6 mice. Neurobiol. Aging 19, 599-606. doi: 10.1016/s0197-4580(98)00098-0

Cavallini, E., Lecce, S., Bottiroli, S., Palladino, P., and Pagnin, A. (2013). Beyond false belief: theory of mind in young, young-old and old-old adults. Int. J. Aging Hum. Dev. 76, 181-198. doi: 10.2190/ag.76.3.a

Chew, J., Gendron, T. F., Prudencio, M., Sasaguri, H., Zhang, Y. J., CastanedesCasey, M., et al. (2015). Neurodegeneration. C9ORF72 repeat expansions in mice cause TDP-43 pathology, neuronal loss, and behavioral deficits. Science 348, 1151-1154. doi: 10.1126/science.aaa9344

Corr, J. (2003). Social behavior in aged rhesus macaques. Coll. Antropol. 27, 87-94.

Davis, M. J., Haley, T., Duvoisin, R. M., and Raber, J. (2012). Measures of anxiety, sensorimotor function, and memory in male and female mGluR4 $4^{-/}$mice. Behav. Brain Res. 229, 21-28. doi: 10.1016/j.bbr.2011.12.037

de Fiebre, N. C., Sumien, N., Forster, M. J., and de Fiebre, C. M. (2006). Spatial learning and psychomotor performance of C57BL/6 mice: age sensitivity and reliability of individual differences. Age 28, 235-253. doi: 10.1007/s11357-0069027-3

Dulac, C. (2006). Sparse encoding of natural scents. Neuron 50, 816-818. doi: 10.1016/j.neuron.2006.06.002

Dulac, C., and Torello, A. T. (2003). Molecular detection of pheromone signals in mammals: from genes to behaviour. Nat. Rev. Neurosci. 4, 551-562. doi: $10.1038 / \mathrm{nrn} 1140$

Enwere, E., Shingo, T., Gregg, C., Fujikawa, H., Ohta, S., and Weiss, S. (2004). Aging results in reduced epidermal growth factor receptor signaling, diminished olfactory neurogenesis, and deficits in fine olfactory discrimination. J. Neurosci. 24, 8354-8365. doi: 10.1523/JNEUROSCI.2751-04.2004

Ey, E., Yang, M., Katz, A. M., Woldeyohannes, L., Silverman, J. L., Leblond, C. S., et al. (2012). Absence of deficits in social behaviors and ultrasonic vocalizations in later generations of mice lacking neuroligin4. Genes Brain Behav. 11, 928-941. doi: 10.1111/j.1601-183x.2012.00849.x

Fahlström, A., Yu, Q., and Ulfhake, B. (2011). Behavioral changes in aging female C57BL/6 mice. Neurobiol. Aging 32, 1868-1880. doi: 10.1016/j.neurobiolaging. 2009.11 .003
Fahlström, A., Zeberg, H., and Ulfhake, B. (2012). Changes in behaviors of male C57BL/6J mice across adult life span and effects of dietary restriction. Age 34, 1435-1452. doi: 10.1007/s11357-011-9320-7

Filiano, A. J., Martens, L. H., Young, A. H., Warmus, B. A., Zhou, P., Diaz-Ramirez, G., et al. (2013). Dissociation of frontotemporal dementiarelated deficits and neuroinflammation in progranulin haploinsufficient mice. J. Neurosci. 33, 5352-5361. doi: 10.1523/JNEUROSCI.6103-11.2013

Fortier, J., Besnard, J., and Allain, P. (2016). Social cognition in normal and pathological aging. Geriatr. Psychol. Neuropsychiatr. Vieil. 14, 438-446. doi: 10.1684/pnv.2016.0640

Frick, K. M., Baxter, M. G., Markowska, A. L., Olton, D. S., and Price, D. L. (1995). Age-related spatial reference and working memory deficits assessed in the water maze. Neurobiol. Aging 16, 149-160. doi: 10.1016/0197-4580(94) 00155-3

Frick, K. M., Burlingame, L. A., Arters, J. A., and Berger-Sweeney, J. (2000). Reference memory, anxiety and estrous cyclicity in C57BL/6NIA mice are affected by age and sex. Neuroscience 95, 293-307. doi: 10.1016/s03064522(99)00418-2

Frick, K. M., Burlingame, L. A., Delaney, S. S., and Berger-Sweeney, J. (2002). Sex differences in neurochemical markers that correlate with behavior in aging mice. Neurobiol. Aging 23, 145-158. doi: 10.1016/s0197-4580(01)00237-8

Gascon, E., Lynch, K., Ruan, H., Almeida, S., Verheyden, J. M., Seeley, W. W., et al. (2014). Alterations in microRNA-124 and AMPA receptors contribute to social behavioral deficits in frontotemporal dementia. Nat. Med. 20, 1444-1451. doi: $10.1038 / \mathrm{nm} .3717$

Guimarães, R. A., Asth, L., Engelberth, R. C., Cavalcante Jde, S., Soares-Rachetti Vde, P., and Gavioli, E. C. (2015). Spontaneous failure of the estrous cycle induces anxiogenic-related behaviors in middle-aged female mice. Physiol. Behav. 147, 319-323. doi: 10.1016/j.physbeh.2015.05.009

Ingram, D. K. (1988). Motor performance variability during aging in rodents. Assessment of reliability and validity of individual differences. Ann. N Y Acad. Sci. 515, 70-96. doi: 10.1111/j.1749-6632.1988.tb32969.x

Ingram, D. K. (2000). Age-related decline in physical activity: generalization to nonhumans. Med. Sci. Sports Exerc. 32, 1623-1629. doi: 10.1097/00005768200009000-00016

Johansson Nolaker, E., Murray, K., Happé, F., and Charlton, R. A. (2018). Cognitive and affective associations with an ecologically valid test of theory of mind across the lifespan. Neuropsychology 32, 754-763. doi: $10.1037 /$ neu0000464

Lalonde, R., and Badescu, R. (1995). Exploratory drive, frontal lobe function and adipsia in aging. Gerontology 41, 134-144. doi: 10.1159/000213674

Lalonde, R., and Strazielle, C. (2009). Exploratory activity and motor coordination in old versus middle-aged C57BL/6J mice. Arch. Gerontol. Geriatr. 49, 39-42. doi: 10.1016/j.archger.2008.04.009

Lamberty, Y., and Gower, A. J. (1990). Age-related changes in spontaneous behavior and learning in NMRI mice from maturity to middle age. Physiol. Behav. 47, 1137-1144. doi: 10.1016/0031-9384(90)90364-a

LaSarge, C. L., Montgomery, K. S., Tucker, C., Slaton, G. S., Griffith, W. H., Setlow, B., et al. (2007). Deficits across multiple cognitive domains in a subset of aged Fischer 344 rats. Neurobiol. Aging 28, 928-936. doi: 10.1016/j. neurobiolaging.2006.04.010

Marck, A., Berthelot, G., Foulonneau, V., Marc, A., Antero-Jacquemin, J., Noirez, P., et al. (2017). Age-related changes in locomotor performance reveal a similar pattern for caenorhabditis elegans, Mus domesticus, Canis familiaris, Equus caballus, and Homo sapiens. J. Gerontol. A Biol. Sci. Med. Sci. 72 455-463. doi: 10.1093/gerona/glw136

Markham, J. A., and Juraska, J. M. (2007). Social recognition memory: influence of age, sex, and ovarian hormonal status. Physiol. Behav. 92, 881-888. doi: 10.1016/j.physbeh.2007.06.020

McQuail, J. A., Frazier, C. J., and Bizon, J. L. (2015). Molecular aspects of age-related cognitive decline: the role of GABA signaling. Trends Mol. Med 21, 450-460. doi: 10.1016/j.molmed.2015.05.002

Moles, A., Costantini, F., Garbugino, L., Zanettini, C., and D’Amato, F. R. (2007). Ultrasonic vocalizations emitted during dyadic interactions in female mice: a possible index of sociability. Behav. Brain Res. 182, 223-230. doi: 10.1016/j.bbr. 2007.01.020

Moran, J. M. (2013). Lifespan development: the effects of typical aging on theory of mind. Behav. Brain Res. 237, 32-40. doi: 10.1016/j.bbr.2012.09.020 
Moran, J. M., Jolly, E., and Mitchell, J. P. (2012). Social-cognitive deficits in normal aging. J. Neurosci. 32, 5553-5561. doi: 10.1523/JNEUROSCI.5511-11.2012

Moreno, M., Richard, M., Landrein, B., Sacquet, J., Didier, A., and Mandairon, N. (2014). Alteration of olfactory perceptual learning and its cellular basis in aged mice. Neurobiol. Aging 35, 680-691. doi: 10.1016/j.neurobiolaging.2013.08.034

Murphy, G. G., Shah, V., Hell, J. W., and Silva, A. J. (2006). Investigation of age-related cognitive decline using mice as a model system: neurophysiological correlates. Am. J. Geriatr. Psychiatry 14, 1012-1021. doi: 10.1097/01.jgp. 0000209404.54310.b3

Patel, R. C., and Larson, J. (2009). Impaired olfactory discrimination learning and decreased olfactory sensitivity in aged C57Bl/6 mice. Neurobiol. Aging 30, 829-837. doi: 10.1016/j.neurobiolaging.2007.08.007

Pettan-Brewer, C., Touch, D. V., Wiley, J. C., Hopkins, H. C., Rabinovitch, P. S., and Ladiges, W. C. (2013). A novel radial water tread maze tracks age-related cognitive decline in mice. Pathobiol. Aging Age Relat. Dis. 3:20679. doi: 10.3402/pba.v3i0.20679

Prediger, R. D., Batista, L. C., and Takahashi, R. N. (2005). Caffeine reverses age-related deficits in olfactory discrimination and social recognition memory in rats. Involvement of adenosine A1 and A2A receptors. Neurobiol. Aging 26, 957-964. doi: 10.1016/j.neurobiolaging.2004.08.012

Rey, N. L., Sacquet, J., Veyrac, A., Jourdan, F., and Didier, A. (2012). Behavioral and cellular markers of olfactory aging and their response to enrichment. Neurobiol. Aging 33, 626.e9-626.e23. doi: 10.1016/j.neurobiolaging.2011. 03.026

Sadoun, A., Rosito, M., Fonta, C., and Girard, P. (2019). Key periods of cognitive decline in a nonhuman primate model of cognitive aging, the common marmoset (Callithrix jacchus). Neurobiol. Aging 74, 1-14. doi: 10.1016/j. neurobiolaging.2018.10.003

Salchner, P., Lubec, G., and Singewald, N. (2004). Decreased social interaction in aged rats may not reflect changes in anxiety-related behaviour. Behav. Brain Res. 151, 1-8. doi: 10.1016/j.bbr.2003.07.002
Shoji, H., Takao, K., Hattori, S., and Miyakawa, T. (2016). Age-related changes in behavior in C57BL/6J mice from young adulthood to middle age. Mol. Brain 9:11. doi: 10.1186/s13041-016-0191-9

Upchurch, M., and Wehner, J. M. (1988a). DBA/2Ibg mice are incapable of cholinergically-based learning in the Morris water task. Pharmacol. Biochem. Behav. 29, 325-329. doi: 10.1016/0091-3057(88)90164-5

Upchurch, M., and Wehner, J. M. (1988b). Differences between inbred strains of mice in Morris water maze performance. Behav. Genet. 18, 55-68. doi: 10.1007/bf01067075

van Schaik, C. P., Isler, K., and Burkart, J. M. (2012). Explaining brain size variation: from social to cultural brain. Trends Cogn. Sci. 16, 277-284. doi: 10.1016/j.tics.2012.04.004

Walton, A., Branham, A., Gash, D. M., and Grondin, R. (2006). Automated video analysis of age-related motor deficits in monkeys using EthoVision. Neurobiol. Aging 27, 1477-1483. doi: 10.1016/j.neurobiolaging.2005.08.003

Wood, E. R., and Dudchenko, P. A. (2003). Aging, spatial behavior and the cognitive map. Nat. Neurosci. 6, 546-548. doi: 10.1038/nn0603-546

Wyss-Coray, T. (2016). Ageing, neurodegeneration and brain rejuvenation. Nature 539, 180-186. doi: 10.1038/nature20411

Conflict of Interest Statement: The authors declare that the research was conducted in the absence of any commercial or financial relationships that could be construed as a potential conflict of interest.

Copyright $\odot 2019$ Boyer, Jaouen, Ibrahim and Gascon. This is an open-access article distributed under the terms of the Creative Commons Attribution License (CC BY). The use, distribution or reproduction in other forums is permitted, provided the original author(s) and the copyright owner(s) are credited and that the original publication in this journal is cited, in accordance with accepted academic practice. No use, distribution or reproduction is permitted which does not comply with these terms. 